\title{
Los procesos de revisión textual en entornos virtuales de aprendizaje. Evaluar para aprender en la universidad
}

\author{
Textual Review Processes in Virtual Learning Environments. Assess to Learn in Higher \\ Education
}

Els processos de revisió textual en entorns virtuals d'aprenentatge. Avaluar per aprendre a la universitat

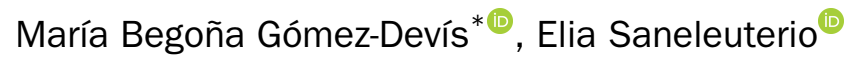 \\ Departamento de Didáctica de la Lengua y la Literatura, Universitat de València, Valencia, España \\ *Autor para correspondencia: m.begona.gomez@uv.es (María Begoña Gómez-Devís)
}

Recibido: 04/11/2019 | Aceptado: 21/04/2020 | Publicado: 24/06/2020

Cómo citar: Gómez-Devís, M.-B.; y Saneleuterio, E. (2020). Los procesos de revisión textual en entornos virtuales de aprendizaje. Evaluar para aprender en la universidad. Research in Education and Learning Innovation Archives, 24,72-88.

10.7203/realia.24.16048

Copyright: El/La Autor/a. Open Access: Este es un artículo de acceso abierto distribuido bajo los términos de la licencia Creative Commons

Attribution-NoDerivatives 4.0 International licence (CC BY-ND 4.0)

\footnotetext{
Financiación: Grup

d'Escriptura Acadèmica i Avaluació en Col-laboració (código:

UV-SFPIE_GER18-847067) y

COLLABORA (código:

UV-SFPIE_PID19-1106445),

financiados por el

Vicerectorat d'Ocupació

i Programes Formatius de la Universitat de València.
}

RESUMEN: Este trabajo presenta los resultados de una investigación vinculada al desarrollo de competencias en evaluación de la expresión escrita - revisión y reescritura- en colaboración y en línea. En ella han participado 167 estudiantes matriculados durante 2018-2019 en primer y cuarto curso de los títulos de grado de Maestro/a en Educación Primaria (EP) y en Educación Infantil (EI) de la Universitat de València. Los datos analizados provienen de la experimentación de una serie de prácticas pedagógicas encaminadas al desarrollo y mejora de las competencias comunicativas académico-profesionales de ambas titulaciones y a todas luces convergentes con los principios del marco metodológico del EEES: (1) la centralidad de las competencias como objeto del aprendizaje universitario; (2) un modelo de aprendizaje orientado a la acción; y (3) un enfoque de la formación universitaria como proceso de acercamiento, incorporación e integración progresivos en la comunidad de expertos y profesionales del área. Para ello, se ha diseñado un procedimiento de evaluación que comprende una serie de tareas focalizadas en la educación lingüística de los futuros maestros, de cuyo análisis emerge el convencimiento del potencial del aprendizaje colaborativo, la eficacia de los entornos virtuales de aprendizaje y de la evaluación orientada al aprendizaje. Como conclusiones más relevantes, se confirma la capacidad del enfoque para generar dinámicas de colaboración entre iguales y su conveniencia para optimizar el aprendizaje de la escritura académica, así como el conocimiento pedagógico de la evaluación

PALABRAS CLAVE: expresión escrita; educación superior; entornos virtuales de aprendizaje; evaluación entre iguales

ABSTRACT: This paper presents the results of a research linked to the skills development in the assessment of written expression-revision and rewriting-in collaboration and online. 167 students have participated, all of them enrolled during 2018-2019 in the first and fourth years of the Master's degrees in Primary Education and Childhood Education of the University of Valencia. The analyzed data come from the experimentation of a series of pedagogical practices aimed at the development and improvement of the academic-professional communication skills of both degrees and clearly convergent with the principles of the EHEA methodological framework: (1) the centrality of the competences as object of university learning; (2) an action-oriented learning model; and (3) a conception of university education as a process of progressive approach, incorporation and integration in the community of experts and professionals in the area. To this aim, an assessment procedure 
has been designed including a series of tasks focused on the linguistic education of future teachers: its analysis emerges the conviction of the potential of collaborative learning, the effectiveness of virtual learning environments and learning-oriented assessment. Some relevant conclusions are confirmed: the ability of the approach to generate collaborative dynamics between peers and its convenience to optimize the learning of academic writing, as well as the pedagogical knowledge of the assessment.

KEYWORDS: written expression; higher education; virtual learning environments; peer assessment

RESUM: Aquest treball presenta els resultats d'una investigació vinculada al desenvolupament de competències en avaluació de l'expressió escrita - revisió i reescriptura- en col-laboració i en línia. Hi han participat 167 estudiants matriculats durant 2018-2019 en primer i quart curs dels títols de grau de Mestre/a en Educació Primària i en Educació Infantil de la Universitat de València. Les dades analitzades provenen de l'experimentació d'una sèrie de pràctiques pedagògiques encaminades al desenvolupament i millora de les competències comunicatives acadèmiques i professionals d'ambdues titulacions i sens dubte convergents amb els principis del marc metodològic de: (1) la centralitat de les competències com a objecte de l'aprenentatge universitari; (2) un model d'aprenentatge orientat a l'acció; i (3) un enfocament de la formació universitària com a procés d'acostament, incorporació i integració progressius en la comunitat d'experts i professionals de l'àrea. Per a això, s'ha dissenyat un procediment d'avaluació que comprèn una sèrie de tasques focalitzades en l'educació lingüística dels futurs mestres: de la seua anàlisi emergeix el convenciment del potencial de l'aprenentatge col-laboratiu, l'eficàcia dels entorns virtuals d'aprenentatge i de l'avaluació orientada a l'aprenentatge. Com a conclusions més rellevants, es confirma la capacitat de l'enfocament per a generar dinàmiques de colllaboració entre iguals i la seua conveniència per a optimitzar l'aprenentatge de l'escriptura acadèmica, així com el coneixement pedagògic de l'avaluació.

PARAULES CLAU: expressió escrita; educació superior; entorns virtuals d'aprenentatge; avaluació entre iguals

\section{Notas de aplicación práctica}

\section{Qué se sabe sobre el tema}

- Desde hace unos años los estudios dedicados a explorar alternativas para mejorar los procesos de aprendizaje colaborativo en entornos virtuales son cada vez más numerosos. En todos ellos se pueden reconocer dos tendencias clave: 1) la aceptación de la mediación colaborativa virtual en los procesos de educación superior y 2) la necesidad de implementar matices metodológicos colaborativos (portafolio y otras alternativas pedagógicas) y ensayar otras herramientas virtuales (redes sociales).

- En el caso concreto de la expresión escrita una de las autoras de este texto publicó recientemente un artículo en el que se revisan las líneas temáticas que desde 2005 hasta 2017 se han publicado sobre Second Language Writing (Selfa-Sastre y Gómez-Devís, 2019)

\section{Qué aporta este trabajo}

- Este artículo aborda una experiencia reciente (curso 2018-19) de aprendizaje de la expresión escrita mediado en entorno virtual, concretamente la plataforma Aula Virtual de la Universitat de València, fruto de la revisión y puesta a punto de experiencias similares en años anteriores. La novedad reside en la descripción de los procesos de planificación, diseño e integración del proyecto de innovación COL·LABORA en la formación universitaria del maestro/a en EP y EI, junto al grado de valoración/percepción de los estudiantes implicados en el proceso de aprendizaje y buenas prácticas.

\section{Implicaciones para la práctica y/o política}

- El artículo presenta una experiencia de innovación educativa centrada en el estudiante universitario que se proyecta sobre el desarrollo de competencias en comunicación (expresión escrita/ escritura académica) y evaluación (formativa y compartida) en entornos virtuales de aprendizaje. Los resultados analizados nos permiten conocer la valoración o grado de percepción de los participantes y ello supone un gran avance para futuras experiencias en la enseñanza superior. 


\section{INTRODUCCIÓN Y MARCO TEÓRICO}

\subsection{Los procesos de escritura en la universidad}

En los últimos años la literatura científica en torno a la expresión escrita ha puesto de manifiesto su naturaleza procesual destacando, especialmente, el papel que tiene respecto del pensamiento. Gracias a ello, y entre otras cosas, encontramos una serie de posiciones teóricas acerca de la escritura y el aprendizaje tales como el uso epistémico de la escritura - critical writing- o escribir para aprender - writing to learn-, que han dado lugar a movimientos orientados hacia la práctica de la escritura a través del currículum o de las disciplinas - writing across the curriculum (WAC) y writing in the disciplines (WID) (Bazerman y Russell, 1994; Britton, 1970; Camps-Mundó y Castelló-Badía, 2013; Russell, 1991)-. Así, observando el uso que durante los procesos de aprendizaje suele hacerse de la escritura, se aprecia que esta cumple una doble función: la primera, comunicativa, transaccional o interpersonal -escribir para interactuar con otras personas o para comunicarnos-; y la segunda, representativa o ideativa -escribir para expresar conocimientos, ideas, sentimientos-. Desde esta segunda función, la escritura se concibe, más allá de la mera expresión de una idea previa, como un instrumento de toma de conciencia, de autorregulación intelectual o de desarrollo y construcción del propio pensamiento.

A pesar de lo consolidado que está el planteamiento de estas investigaciones entre los especialistas en alfabetización académica, indagar el entramado procesual de la escritura, repensar las condiciones pedagógicas en las que pedimos escribir al alumnado en las distintas etapas educativas o atender la naturaleza de la lengua con la que se escribe - L1, L2 o LE- son retos a los que todavía no se ha dado suficiente atención en la práctica docente de las disciplinas no lingüísticas (Castelló, 2014; Hurtado-González, 2019). Ciñéndonos al ámbito de la educación superior, los estudios dedicados a la composición escrita han establecido numerosos escollos sobre los que hay que seguir actuando. En palabras de Carlino (2004) pueden articularse en torno a:

1. la dificultad para escribir teniendo en cuenta la perspectiva del lector;

2. el desaprovechamiento del potencial epistémico de la escritura;

3. la propensión a revisar los textos solo en forma lineal y centrándose en aspectos locales y poco sustantivos;

4. la dilación o postergación del momento de empezar a escribir.

Al observarlas en el aula universitaria, por separado o en conjunto, y siendo conscientes de los beneficios del aprendizaje colaborativo en todas las etapas educativas $-\mathrm{y}$ especialmente si se optimiza con herramientas tecnológicas (García-Chitiva y SuárezGuerrero, 2019)-, es palmaria la necesidad de innovación a través de la activación de prácticas o tareas enfocadas hacia la colaboración entre estudiantes - o entre discentes y docente- en las que no solo la escritura, sino también la revisión o valoración de lo escrito incidan en una formación integral del alumnado (Bustos-Sánchez, 2009; Erkens, Jaspers, Prangsma, y Kanselaar, 2005; Saneleuterio y García-Ramos, 2015).

\subsection{El aprendizaje colaborativo y la escritura en línea}

Los enfoques colaborativos sintonizan con algunas de las bases que promueve el Espacio Europeo de Educación Superior (EEES), especialmente el aumento de protagonismo del rol discente y la consecuente superación de la figura docente como agente principal en la transmisión y evaluación del aprendizaje. Por ello, las instituciones educativas promueven, cada vez más, el valor añadido que representa fomentar que el alumnado aprenda con los otros y de los otros buscando la forma en que cada estudiante cuente con oportunidades de aprender en grupo colaborando con sus pares para resolver 
problemas auténticos que supongan un uso situado del conocimiento. Se recomienda, en consecuencia, aprender a colaborar, es decir, escuchar las opiniones de los otros, considerarlas, buscar acuerdos y gestionar juntos las soluciones generadas por el grupo.

De hecho, la alta valoración del aprendizaje colaborativo en la Educación Superior en entornos virtuales de aprendizaje (EVA) se ha visto nutrida por líneas de investigación recientes que se interesan en analizar y comprender cómo la colaboración y la tecnología pueden favorecer la construcción del conocimiento y cómo el aprendizaje colaborativo mediado tecnológicamente, bien orientado, en efecto optimiza la interacción y el trabajo en grupo (García-Chitiva y Suárez-Guerrero, 2019; Scardamalia y Bereiter, 2003, 2006).

En el marco de la producción textual, Erkens et al. (2005) sugieren que la principal ventaja de la escritura colaborativa, comparada con la individual, es la posibilidad de recibir y ofrecer retroalimentación inmediata y argumentativa.

No obstante, si bien es evidente que desde hace unos años la tecnología ha tomado un papel relevante en el mundo educativo, pues implica flexibilidad para aplicar instrumentos variados y adaptados al contexto o necesidades del alumnado, su introducción en la actividad universitaria no representa un cambio innovador per se. De hecho, todavía queda pendiente el reto de acompasar el rol de docente y estudiantes, la metodología y el sistema de evaluación; en otras palabras, la investigación que ahora se aborda busca experimentar e indagar el desarrollo de propuestas tecno-pedagógicas para aprender escribiendo con iguales o para aprender solucionando problemas con los otros mediante la propia escritura en línea - online collaborative writing - o a través de la revisión, también mediante herramientas virtuales, de textos escritos por compañeros y compañeras. Entre las numerosas opciones tecnológicas para el soporte de los procesos de escritura en línea se destacan dos, ambas válidas para las fases de planificación, textualización y revisión:

- Wiki, que puede concebirse como una aplicación de informática colaborativa que permite crear colectivamente documentos web usando un simple esquema de etiquetas y marcas, y que no requiere de una instancia jerárquica que apruebe la publicación de las modificaciones (Piscitelli, 2005, 87).

- Entornos para la construcción del conocimiento -knowledge building environment-, los cuales parten del supuesto teórico de Scardamalia y Bereiter (2006) y plantean la construcción del conocimiento a través de un proceso de creación resultado de compartir objetivos, discutir de modo grupal y sintetizar las ideas.

\subsection{La colaboración en la evaluación y su orientación hacia el aprendizaje}

Finalmente, el marco teórico del que parte este estudio tiene que ver con lo que se conoce como evaluación orientada al aprendizaje (EOA) - Learning Oriented Assessment en inglés (Carless, 2007, 2009; Carless, Joughin, y Mok, 2006)-. El objetivo del enfoque quiere trascender la mera valoración del aprendizaje, busca impulsarlo y optimizarlo, y para ello hay que "desarrollar la autonomía del estudiante, el aprendizaje permanente y su responsabilidad para orientar y gestionar su propio proceso de aprendizaje aprendizaje estratégico-" (Ibarra y Rodríguez-Gómez, 2010, p. 446). También este cambio de perspectiva implica un profesorado dispuesto a diseñar procedimientos y utilizar métodos que favorezcan el protagonismo del alumnado y su participación en la toma de decisiones.

Así, las claves del entramado de la EOA son las siguientes Ibarra y Rodríguez-Gómez (2010); Reyes-García, Marchena-Gómez, y Sosa-Moreno (2012): entender las tareas de evaluación como tareas de aprendizaje; garantizar que exista una retroalimentación adecuada que resulte útil para mejorar en tareas posteriores; favorecer la implicación 
de cada estudiante en sus procesos de evaluación y en los de sus iguales para una mejor comprensión de las metas de aprendizaje y un trabajo más activo hacia el logro de los estándares y criterios. Finalmente, cabe añadir la necesidad de no olvidar el potencial didáctico de las TIC (Gros, 2015), nuevas formas de acción educativa y de representación pedagógica, también para la evaluación, a modo de lo que se ha denominado e-EOA (Gómez-Devís y Saneleuterio, 2015; Gómez-Ruiz, Rodríguez-Gómez, y IbarraSáiz, 2013; Ramos, Chiva, y Gómez-Devís, 2017; Rodríguez-Gómez y Ibarra-Saiz, 2015).

\section{PROPÓSITO Y METODOLOGÍA}

Este trabajo se enmarca en los modelos metodológicos de la investigación-acción en el ámbito educativo y forma parte de un proyecto de innovación docente que, aunque basado en experiencias previas (Gómez-Devís, 2015; Gómez-Devis, Garcia-Raffi, y Saneleuterio, 2017; Ramos et al., 2017; Saneleuterio y García-Ramos, 2015), propiamente comenzó el curso 2018-19. Cada renovación en los programas de innovación de la propia institución ha supuesto mejoras pedagógicas respecto del precedente, ora derivadas del análisis de la práctica observada, ora de la incorporación de nuevos instrumentos de recogida de datos que permiten aproximarnos mejor al objeto de estudio.

\subsection{El reto de la revisión textual colaborativa en la Universitat de València}

Desde la perspectiva expuesta en el marco teórico y con una amplia experiencia en el marco de los programas de innovación educativa de la Universitat de València de los últimos años, se presentan los resultados obtenidos en torno al diseño y experimentación de una práctica de expresión escrita - reescritura y revisión colaborativa en línea- y su evaluación entre iguales.

Dicha propuesta didáctica reúne las tesis de escritura y evaluación como herramientas para aprender y para ello se ha implementado una metodología activa acorde con el seguimiento de un proceso de aprendizaje colaborativo: planificación en pareja, escritura individual, con revisión y evaluación compartidas, a lo largo de un diario virtual que recoge las actas de las sesiones presenciales de cuatro grupos de dos asignaturas de los grados en Maestro/a en Educación Infantil y Maestro/a en Educación Primaria ${ }^{1}$.Tampoco hay que olvidar el hecho de que el contenido de los textos que deben revisarse y reescribirse corresponde a los apuntes de la asignatura; en consecuencia, se promueve con ello la figura del lector crítico que conoce y atiende a las indicaciones de la comunidad académica a la que pertenece (Russell y Cortés, 2012).

En definitiva, este proyecto de innovación se originó como una estrategia para favorecer, desde el primer al último curso de las titulaciones de Magisterio, una mejora sustancial de la competencia en la composición de textos cuyo sentido y significado está vinculado a elaborar, construir y reflexionar sobre el conocimiento, y dar cuenta de lo aprendido.

\subsection{Instrumentos, materiales y participantes}

Tras unos años de pilotaje, los instrumentos empleados en la actualidad para la mejora de la expresión escrita -y especialmente centrados en la escritura y revisión- son el foro virtual (borrador del acta, comentarios de revisión inter pares, acta definitiva y rúbricas de evaluación argumentadas), un cuestionario de autoevaluación y un cuestionario de satisfacción, también virtuales. Los participantes del presente estudio son

\footnotetext{
${ }^{1}$ Las asignaturas implicadas son Lengua Española para Maestros, de primer curso, y Planificación de la Lengua y la Literatura, de cuarto curso.
} 
167 estudiantes de primer y cuarto curso de los grados de Maestro/a en Educación Infantily en Educación Primaria organizados en grupos naturales de cuarenta alumnos aproximadamente (Tabla 1).

Tabla 1. Grupos naturales de los participantes. Fuente: elaboración propia

\begin{tabular}{|c|c|c|c|c|}
\hline Año académico & Curso & Asignatura & Código & $\begin{array}{l}\text { Estudiantes } \\
\text { matriculados }\end{array}$ \\
\hline 2018-19 & $1 .^{\circ}$ & $\begin{array}{l}\text { Lengua } \\
\text { Española para } \\
\text { Maestros }\end{array}$ & 33606 & 43 \\
\hline $2018-19$ & $1 .^{\circ}$ & $\begin{array}{l}\text { Lengua } \\
\text { Española para } \\
\text { Maestros }\end{array}$ & 33606 & 36 \\
\hline $2018-19$ & $4 .^{\circ}$ & $\begin{array}{l}\text { Planificación } \\
\text { de la Lengua y } \\
\text { la Literatura }\end{array}$ & 33660 & 45 \\
\hline 2018-19 & $4 .^{\circ}$ & $\begin{array}{l}\text { Planificación } \\
\text { de la Lengua y } \\
\text { la Literatura }\end{array}$ & 33660 & 43 \\
\hline
\end{tabular}

Los miembros del equipo docente e investigador son conscientes de que la tendencia mayoritaria de los futuros maestros y maestras a la hora de revisar un texto, ajeno o propio, consiste en señalar básicamente las faltas de ortografía y los errores de referencia o contenido; hecho coherente con estudios como el de Ruiz-Flores (2009), quien señala que las anotaciones del profesorado en activo también suelen centrarse en errores puntuales de gramática y ortografía. Dicho esto, en el contexto de una materia universitaria del Departamento de Didáctica de la Lengua y la Literatura debe evidenciarse la insuficiencia o superficialidad de estas tendencias de corrección focalizadas en el uso del código, ya que invitan a pensar que estos aspectos constituyen lo más importante del texto escrito.

\subsection{Objetivos}

De acuerdo con esta premisa, el principal propósito de esta investigación-acción vinculada al contexto universitario consiste en dotar de estrategias que trasciendan la mera corrección ortográfica y gramatical en la revisión de textos, y así ahondar en cuestiones de estructura, sentido o adecuación al género.

A partir de breves diálogos en las sesiones presenciales entre profesor y grupo de estudiantes se pretendía mediar en la fase de revisión aportando ejemplos sobre cómo atender aspectos que trasciendan la revisión superficial. Este feedback no se produjo de manera escrita, ni con anotaciones en los borradores publicados, ni mediante intervenciones en el foro - que se dejaba íntegramente a disposición del alumnado-, sino que se dedicaron a revisar en gran grupo -autores, revisores y docente- los textos y comentarios publicados. Tal como se ha apuntado en investigaciones similares MartínezLorca y Zabala-Baños (2015), esta dinámica ayuda a descubrir fortalezas o debilidades del proceso, regular el estilo expresivo propio, dotar de ciertas garantías las primeras incursiones textuales y favorecer la toma de conciencia para que la articulación entre intuición y reflexión sea esencial en el uso escrito de la lengua.

Para el diseño de la intervención se consideraron los beneficios de la escritura en colaboración no solo porque ayuda a explicitar estrategias de redacción diferentes según la fase de composición por parte de cada participante, sino porque genera dinámicas que no se producen en la escritura individual (Âlvarez Angulo, 2011). De entre ellas priorizamos aquellas que se producen durante las fases de revisión, especialmente fructíferas en la mejora de la escritura académica en contextos universitarios (BustosSánchez, 2009; Hurtado-González, 2019; Martínez-Lorca y Zabala-Baños, 2015), muy 
relacionadas con los procesos de evaluación de la escritura y muy oportunas, por tanto, en la formación de maestros (Gómez-Devis et al., 2017; Tapia, 2017).

Así pues, la presente aportación tiene el objetivo de indagar qué aspectos son percibidos por los estudiantes implicados como puntos fuertes y cuáles perciben como mejorables en relación con la tarea de escritura académica y sus procesos de enseñanzaaprendizaje y evaluación. Concretamente, este objetivo general se desgrana en tres objetivos específicos: (1) saber si la elaboración del diario y las conversaciones en el foro resultan útiles para mejorar la expresión escrita en el ámbito académico y para llevar la asignatura al día; (2) valorar la relación entre el esfuerzo y los beneficios de la tarea; (3) conocer la satisfacción del alumnado respecto de los procesos de evaluación experimentados.

\subsection{Diseño de la propuesta didáctica}

El producto final de la tarea de escritura es la creación colectiva de un diario virtual que contiene los informes de todas las sesiones presenciales de la asignatura. Durante su elaboración se requiere una revisión colectiva y compartida de cada texto -borradorantes de su publicación definitiva en la plataforma Aula Virtual. Así, además de que el resultado global es cooperativo - cada estudiante se encarga de una sesión, pero puede observar y participar en la mejora de los informes ya publicados en el foro-, lo que verdaderamente es colaborativo es el proceso de revisión, de manera que cada texto individual incorpora, en realidad, las sugerencias que los compañeros y compañeras han ido apuntando de manera argumentada.

Con objeto de favorecer el funcionamiento fluido del foro -diario virtual- se consensuaron algunas consignas de modo que el conjunto de textos definitivos fuera lo más homogéneo posible y, además, que todos los estudiantes desempeñaran correctamente sus roles y ajustaran su nivel de participación a lo requerido. Son las siguientes:

1. Cada sesión de aula se aborda normalmente por dos secretarios o secretarias; ello obedece al número habitual de estudiantes por grupo y su correspondencia con el número de sesiones de la asignatura durante un cuatrimestre.

2. El reparto de sesiones corre a cargo del grupo de estudiantes, así como su gestión, con el establecimiento de los criterios o procedimientos que el mismo determine.

3. El canal de comunicación exclusivo para todo el proceso relativo al diario de sesiones es la plataforma virtual de la universidad (Moodle).

4. Cada estudiante, cuando ejerce como secretario o secretaria, debe enviar el mismo día de la sesión presencial una primera versión del informe de manera individual. La planificación del texto puede elaborarse con el otro compañero que vaya a encargarse de la misma sesión.

5. Las revisiones o propuestas de mejora se hacen a través de mensajes contextualizados que se publican en el mismo hilo del foro donde se ha publicado el primer borrador.

6. La versión definitiva es responsabilidad individual del autor o autora. Esta debe completarse y publicarse a los quince días de la primera publicación -borrador-, incorporando las revisiones oportunas de quienes hayan participado en la conversación virtual y contestando en el foro a aquellas que no se han considerado adecuadas.

7. Al acabar el cuatrimestre, cada estudiante evalúa el desempeño de, al menos, cuatro compañeros o compañeras, sirviéndose de una rúbrica de evaluación proporcionada para tal fin. 
8. Por último, se acuerda la autoevaluación de la tarea mediante un cuestionario virtual que contempla dos procesos: la reescritura del acta individual -borrador y versión definitiva - y la participación tanto en la revisión de algunos de los de textos elaborados por los compañeros como en su evaluación.

Desde nuestra posición, la decisión de utilizar el foro alojado en el Aula Virtual institucional y no otros soportes, como pueda ser una wiki o un documento compartido, reside en que cada revisión debe justificarse, no solo "corregirse", y este formato permite que el autor responda, matice o justifique lo que considere oportuno. Del mismo modo, su carácter asincrónico, limitado en un periodo de quince días, permite ampliar tanto el número de revisores como el tiempo que cada uno necesite en la reflexión y valoración del texto ajeno. Se trata, pues, de una estrategia pedagógica que promueve los procesos de lectura crítica. Esto es así porque, por un lado, es muy probable que verdaderamente al lector o lectora le interese qué se dice ya que va a tener que examinarse de toda la materia; y, por otro, le interesará desarrollar la capacidad de identificar los géneros textuales y los usos que se dan en la enseñanza de las disciplinas.

Por último, este proceso de e-evaluación orientada al aprendizaje garantiza que el estudiante se convierta en protagonista de su propio aprendizaje poniendo en práctica estrategias metacognitivas que ayuden a la autorregulación, reflexionando sobre su pensamiento y comparándolo con el de los otros, a través de la autoevaluación y la evaluación entre iguales. Asimismo, mitiga la tendencia tradicional del docente a monopolizar la actividad de enseñar y evaluar, limitando su responsabilidad a la facilitación de los instrumentos y criterios de evaluación, así como al control de la honestidad mediante sistema de auditoría, sin olvidar el rol principal y que dota de sentido a su actividad, el de funcionar de guía accesible durante todo el proceso.

\subsection{Cuestionario de autoevaluación y percepción}

El proceso de escritura, revisión y reescritura del diario virtual de sesiones concluye con una propuesta de evaluación participativa en que cada estudiante valora el desempeño de otros compañeros o compañeras y, finalmente, se autoevalúa mediante un cuestionario que incluye diez preguntas de percepción y valoración tanto de la tarea como del diseño del procedimiento de evaluación. Las respuestas de las nueve primeras se organizan en una escala Lickert graduada en cinco puntos (desde "Totalmente de acuerdo" hasta "Totalmente en desacuerdo") y la pregunta final queda abierta para comentarios. Por cuestiones de extensión nos centraremos únicamente en aquellas que tienen que ver con los objetivos específicos de este artículo, las cuales se relacionan en la Tabla 2.

\section{ANÁLISIS Y DISCUSIÓN DE LOS RESULTADOS}

Antes de proseguir con el análisis de las percepciones y nivel de satisfacción de los futuros maestros, es necesario señalar que, en una fase de exploración previa, los profesores implicados en la investigación detectaron, como debilidades representativas de los textos publicados en el foro, los siguientes aspectos: falta de planificación previa o planificación deficiente, errores en el uso de la información o en el desarrollo del contenido, dominio insuficiente de la norma que provoca errores de uso sistemáticos, cierta dependencia de lo literal - es decir, de lo escuchado durante la clase-, escasa atención o falta de hábitos para la revisión, etc. Asimismo, se señala que las revisiones efectuadas entre iguales tampoco atienden todas estas deficiencias estructurales, especialmente las correspondientes a las primeras sesiones del cuatrimestre. Aun así, y teniendo en cuenta que el número de revisores varía en función del grupo observado y en cada uno de los textos publicados, se percibe mayor calado e intensidad en las aportaciones realizadas en el periodo final de la asignatura. 
Tabla 2. Preguntas sobre la percepción y valoración de los participantes. Fuente: elaboración propia.

\begin{tabular}{|c|c|}
\hline Pregunta 1 & $\begin{array}{l}\text { La elaboración del diario y las conversaciones en el foro me han resultado } \\
\text { útiles para mejorar mi expresión escrita en el ámbito académico }\end{array}$ \\
\hline Pregunta 2 & $\begin{array}{l}\text { En mi opinión, incluso los peores informes fueron mejorados } \\
\text { notablemente en su versión definitiva }\end{array}$ \\
\hline Pregunta 3 & $\begin{array}{l}\text { Considero que el foro virtual es una herramienta adecuada para la } \\
\text { revisión colectiva de textos }\end{array}$ \\
\hline Pregunta 4 & He consultado el diario de sesiones con cierta regularidad \\
\hline Pregunta 5 & $\begin{array}{l}\text { Los informes publicados en el diario de sesiones me han resultado útiles } \\
\text { para llevar la asignatura al día }\end{array}$ \\
\hline Pregunta 6 & $\begin{array}{l}\text { El esfuerzo dedicado ha merecido la pena considerando los beneficios } \\
\text { obtenidos }\end{array}$ \\
\hline Pregunta 7 & $\begin{array}{l}\text { Para esta actividad me parece adecuado el sistema de evaluación entre } \\
\text { iguales }\end{array}$ \\
\hline Pregunta 8 & $\begin{array}{l}\text { Todas las evaluaciones recibidas me parecen justas dado que mis } \\
\text { compañeros han justificado sus decisiones }\end{array}$ \\
\hline Pregunta 9 & $\begin{array}{l}\text { En general, he quedado satisfecho/a con la metodología de esta tarea y } \\
\text { la recomendaría. }\end{array}$ \\
\hline
\end{tabular}

A continuación, presentamos los valores obtenidos en cada una de las preguntas del cuestionario seleccionadas para este estudio.

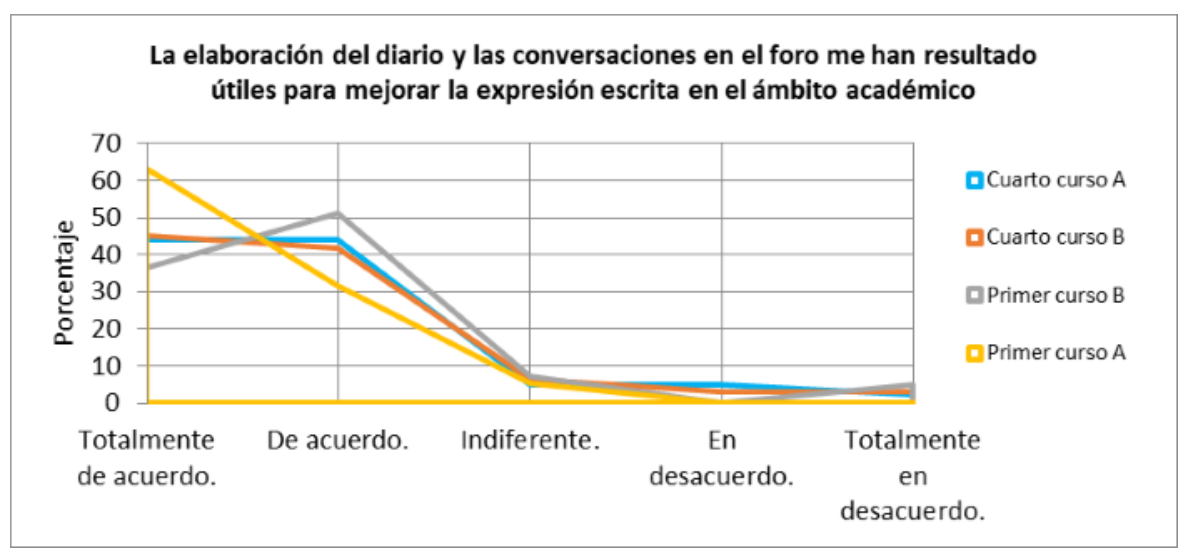

Gráfico 1: Respuestas de los estudiantes de primer y cuarto curso a la pregunta 1. Fuente: elaboración propia

Como se observa en elGráfico 1, prácticamente la totalidad de estudiantes se muestra de acuerdo o totalmente de acuerdo con la afirmación de la pregunta 1. Aunque se aprecia cierta disparidad de matices en los dos subgrupos de primer curso, es posible distinguir valores muy semejantes en cuarto curso. En todo caso, los resultados nos permiten afirmar que el alumnado valora los procesos de escritura y discusión en el foro virtual como útiles para mejorar su expresión escrita en el ámbito académico.

En elGráfico 2 sin embargo, los alumnos y alumnas se muestran más críticos a la hora de valorar la implicación en la actividad de la totalidad de compañeros, pues son conscientes de que no todos los informes fueron mejorados "notablemente" en la versión definitiva. Ello explica que entre las respuestas encontremos participantes reacios o reacias a marcar la opción "totalmente de acuerdo", e incluso que una parte considerable se muestre indiferente.

Respecto a las posibilidades de la herramienta de revisión textual, la percepción del alumnado es casi unánime: el foro virtual es valorado como adecuado o muy adecuado, 


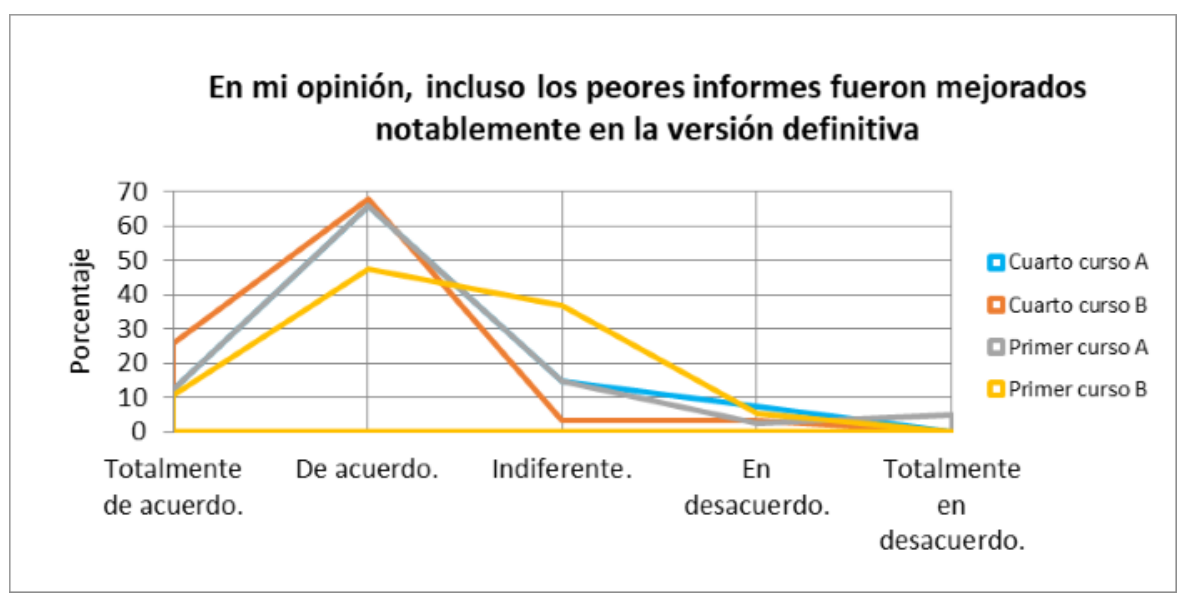

Gráfico 2: Respuestas de los estudiantes de primer y cuarto curso a la pregunta 2. Fuente: elaboración propia

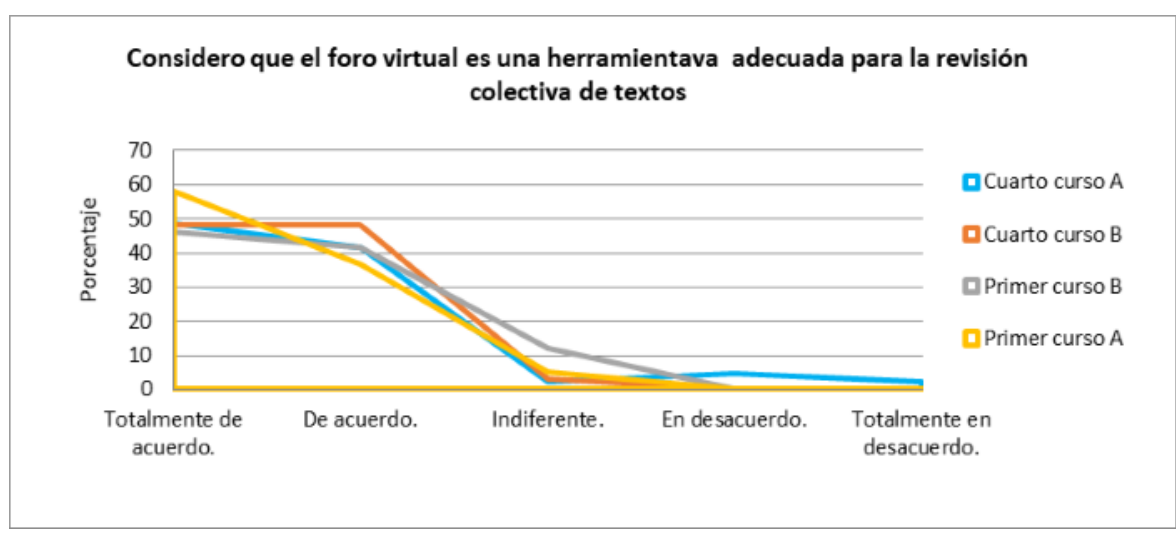

Gráfico 3: Respuestas de los estudiantes de primer y cuarto curso a la pregunta 3. Fuente: elaboración propia

según queda implícito en sus respuestas a la tercera cuestión (Gráfico 3). Estos resultados confirman nuestra decisión de utilizar este sistema de conversación asincrónica frente a las alternativas barajadas, como la wiki o los documentos compartidos, quizás más útiles para cuando el número de revisores es menor o para cuando el componente colaborativo comienza en la fase de textualización.

No existen investigaciones que midan la percepción del estudiantado con este tipo de recursos cuando se los orienta específicamente a la tarea de revisión textual; no obstante, en la evaluación que aporta Tapia (2017) se percibe que, siendo voluntaria, sí es alta la participación virtual en este tipo de foros de discusión. Además, nuestros resultados están en sintonía con otros estudios que evalúan la eficacia del aprendizaje combinado (Solano-Córdoba, 2013) o el potencial de los foros para el aprendizaje de un idioma (Cruz-Piñol, 2014).

Los Gráficos 4 y 5, por su parte, nos muestran la importancia de esta actividad en el trabajo diario del alumnado y su utilidad para llevar la asignatura al día. Salvo el subgrupo de primero A, que responde muy positivamente a esta segunda cuestión, los resultados son muy parecidos en ambas preguntas: la consulta del diario ha tenido lugar con cierta regularidad y los informes elaborados por iguales han ayudado a llevar una continuidad en el estudio.

Esta sensación de utilidad percibida por los estudiantes es más valiosa si tenemos en cuenta que la elaboración del diario de sesiones no comporta una sobrecarga de trabajo de escritura, dado que cada uno solamente ha de encargarse de una sesión de 


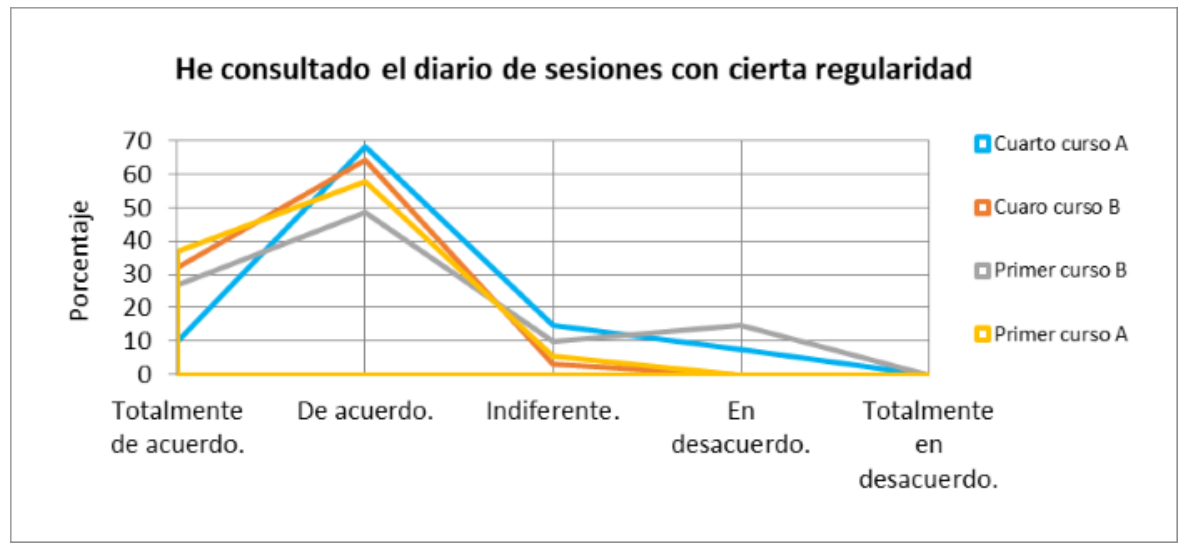

Gráfico 4: Respuestas de los estudiantes de primer y cuarto curso a la pregunta 4. Fuente: elaboración propia

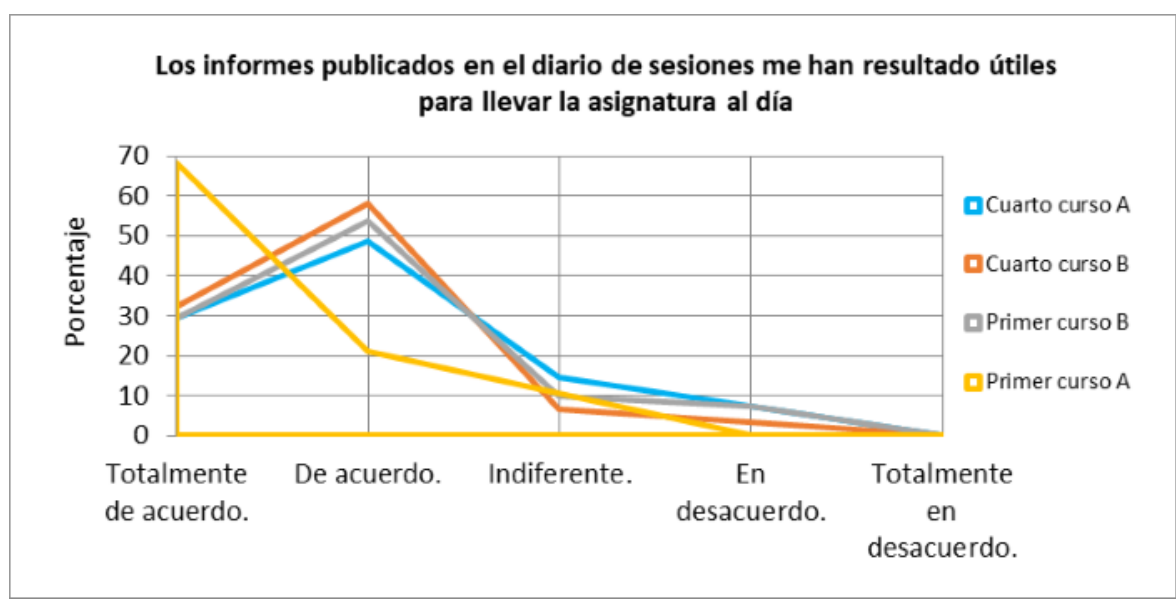

Gráfico 5: Respuestas de los estudiantes de primer y cuarto curso a la pregunta 5. Fuente: elaboración propia.

la asignatura. No obstante, cabe señalar que sí implica una participación continua de lectura y revisión de los textos de los compañeros, escritura de mensajes de retroalimentación y, finalmente, un esfuerzo añadido de autoevaluación y de evaluar a algunos compañeros. Por ello, nos parecía fundamental conocer el balance esfuerzo-beneficios desde la perspectiva del alumnado. Como se muestra en el Gráfico 6, la curva de percepción es similar en los cursos analizados: entre un $75 \%$ y un $90 \%$ de estudiantes, según los grupos, se decantan por las opciones "de acuerdo" y "totalmente de acuerdo" con la idea de que el esfuerzo ha merecido la pena.

Una de nuestras mayores preocupaciones ha sido desde el principio mitigar la insatisfacción o recelo del alumnado respecto la evaluación entre iguales. A lo largo de las diferentes ediciones del proyecto se ha insistido mucho en este aspecto, siempre con el objetivo de evitar agravios comparativos y poder asegurar una evaluación justa, al tiempo que provechosa pedagógicamente. Las respuestas a las preguntas 7 y 8 abordan este aspecto. Respecto al sistema de evaluación entre iguales (Gráfico 7), apenas se registraron respuestas negativas en este sentido, si bien sí encontramos entre un $10 \%$ y un $15 \%$ de estudiantes indiferentes.

La pregunta 8, por su parte, es en la que se registra mayor disparidad de respuestas (Gráfico 8). A tenor de los resultados, los estudiantes del último curso de magisterio perciben de modo distinto la competencia evaluadora de sus compañeros. Mientras que los de primer curso se encuentran relativamente satisfechos con la evaluación 


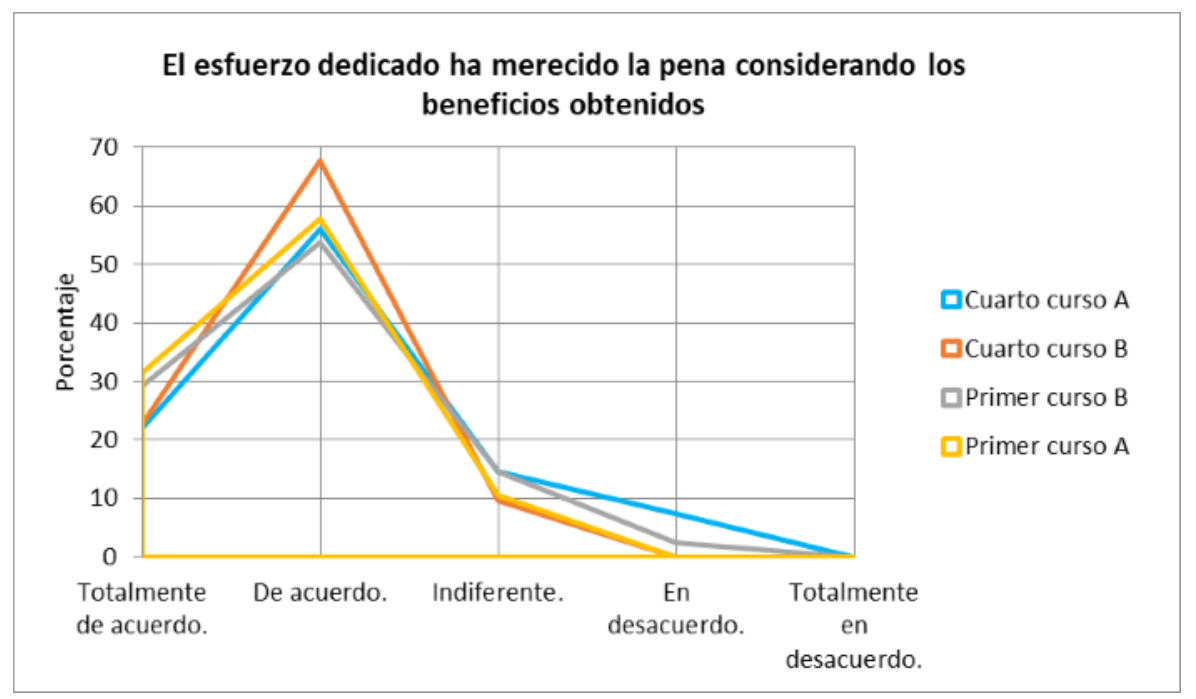

Gráfico 6: Respuestas de los estudiantes de primer y cuarto curso a la pregunta 6. Fuente: elaboración propia.

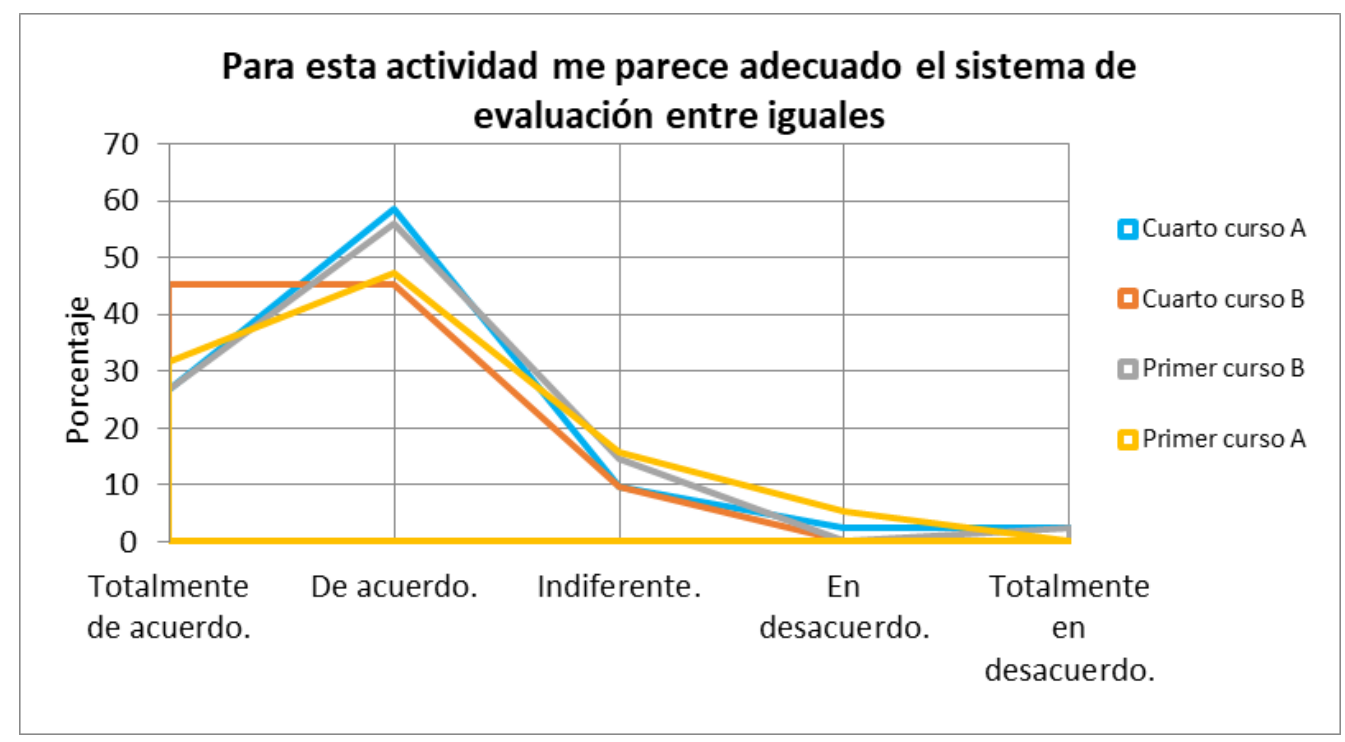

Gráfico 7: Respuestas de los estudiantes de primer y cuarto curso a la pregunta 7. Fuente: elaboración propia.

recibida, los de cuarto se muestran mucho más críticos, quizás porque a través de su propia experiencia en los estudios del grado conciben la evaluación en su complejidad y esperan ciertos matices y consideraciones a la hora de ser evaluados, por un lado, y por otro, tal vez la contemplación de estos matices por parte de sus iguales implica haber recibido calificaciones más bajas. No obstante, es necesario afinar esta cuestión en próximas ediciones a fin de recabar información más precisa y extraer conclusiones más rigurosas.

Como discusión general de los resultados relativos a la satisfacción del estudiantado con los procesos de evaluación experimentados, estos son más bajos que los datos presentados por investigaciones recientes que miden procesos parecidos, incluso en la formación de maestros, donde se percibe la competencia de saber evaluar como clave para el futuro ejercicio docente (Gómez-Ruíz y Quesada-Serra, 2017; RodríguezEspinosa, Restrepo-Betancur, y Luna-Cabrera, 2016; Sosa-Alonso, Santos-Vega, VegaNavarro, y Marrero-Acosta, 2018). Quizás ello se deba a que nuestro estudio, a dife- 


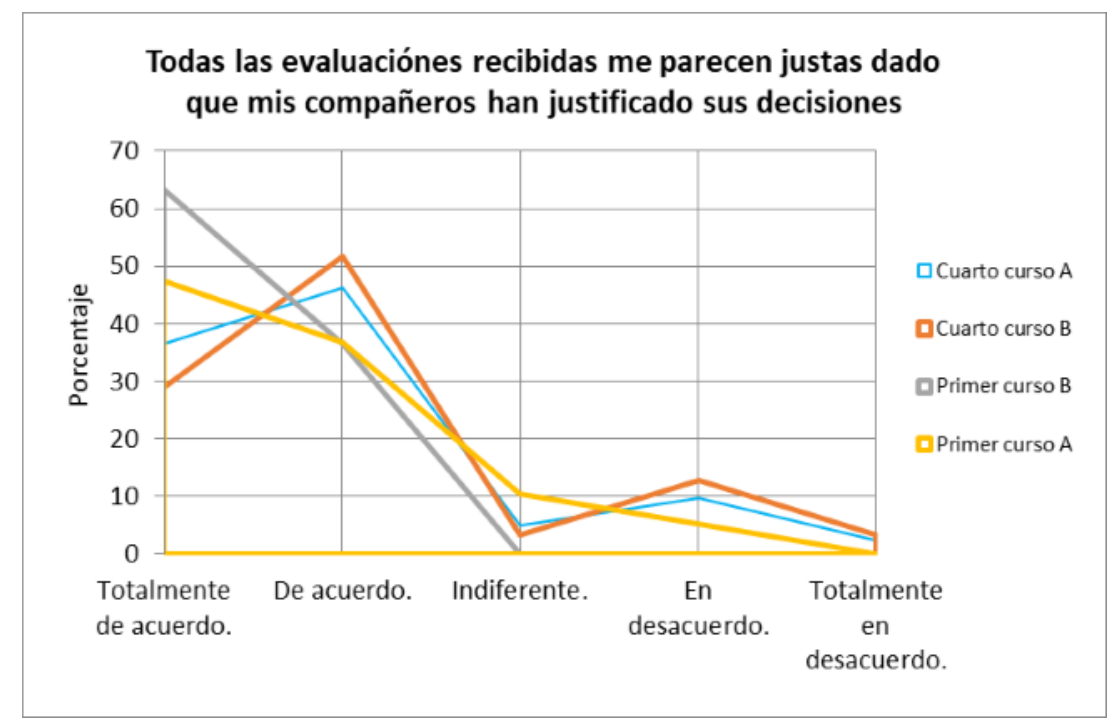

Gráfico 8: Respuestas de los estudiantes de primer y cuarto curso a la pregunta 8. Fuente: elaboración propia.

rencia de los citados, incluye grupos de estudiantes de último curso, que generalmente presentan características muy particulares que los distinguen del resto de cursos de la titulación y con expectativas más altas en cuanto a la evaluación.

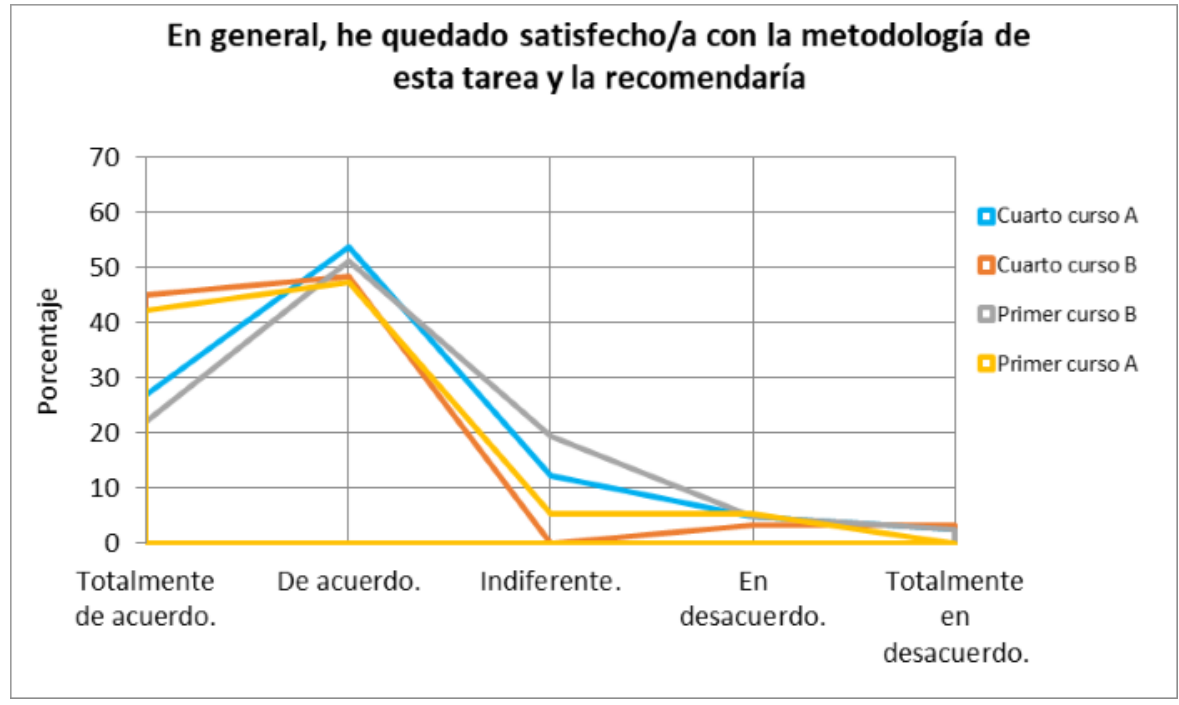

Gráfico 9: Respuestas de los estudiantes de primer y cuarto curso a la pregunta 9. Fuente: elaboración propia.

Finalmente, la valoración general de la tarea (Gráfico 9) devuelve un balance algo diferente según los subgrupos, pero en todo caso positivo para la mayoría de los estudiantes implicados -en 4A $(80 \%)$ y $1 \mathrm{~B}(73 \%)$ - o para la inmensa mayoría -en $4 \mathrm{~B}(94 \%)$ y $1 \mathrm{~A}(90 \%)-$.

A modo de cierre, y en la línea de la discusión de gráficos anteriores, al comparar estos resultados con la satisfacción que, en general, presenta la introducción de metodologías activas (Sosa-Alonso et al., 2018; Vicent, Gonzálvez, Sanmartín, y MartínezMonteagudo, 2018) es posible afirmar que nuestra investigación-acción avanza en la dirección adecuada. 


\section{CONCLUSIONES}

La actividad de coordinación entre personas que buscan aprender no es sencilla de gestionar y su complejidad aumenta cuando en la actividad colaborativa surge un nuevo componente, la tecnología. Ciñéndonos a los datos del apartado anterior, aunque preliminares puesto que corresponden a una parte del total de participantes en el proyecto de innovación 2018-19 -167 estudiantes repartidos en cuatro grupos naturales-, es posible obtener algunas conclusiones sobre las características del desarrollo de una investigación centrada en los procesos de escritura y revisión textual colaborativa y en línea en la universidad.

La primera tiene que ver con el objetivo de conocer si la elaboración del diario y las conversaciones en el foro resultan útiles para mejorar la expresión escrita en el ámbito académico y para llevar la asignatura al día. Tal y como se ha comprobado (Gráficos 1, 3 y 5), los participantes perciben que el entorno virtual utilizado para la elaboración del diario junto al registro de las conversaciones mantenidas ha sido adecuado no solo para llevar la asignatura al día, sino también para los procesos de revisión colectiva de textos.

Asimismo, y ligado al segundo objetivo, el desarrollo de esta tarea es una clara apuesta en favor de la evaluación orientada al aprendizaje. Antes de la publicación en el foro del texto final -informe definitivo- ha tenido lugar la revisión colectiva de un texto borrador-, la cual, una vez subsanados los errores derivados del dominio insuficiente del código, el desarrollo de destrezas en detección y diagnóstico de problemas con el contenido y la estructura de distintos textos, ha propiciado versiones personales notablemente mejoradas con respecto a la mayoría de producciones anteriores (Gráficos 2 y 4). Hecho que también se corrobora al observar el balance entre esfuerzo y beneficios (Gráfico 6).

El tercer y último objetivo atiende la satisfacción de los participantes en relación con los procesos de e-evaluación experimentados. De acuerdo con los resultados recabados (Gráficos 7 y 8) tanto autores como revisores se muestran satisfechos con su participación en la evaluación -entre iguales- y con la calificación obtenida; si bien en este último aspecto distingue a los estudiantes de cuarto curso como más críticos que los de primero.

Finalmente, cabe no descuidar el hecho de que los procesos de evaluación compartida aumentan las estrategias y capacidades de los maestros y maestras en formación para el futuro ejercicio de su profesión. El análisis de si son así percibidas por nuestros estudiantes es una de las prospectivas que cabe transitar en esta línea de investigación.

Por último, se ha dispuesto la pregunta final (Gráfico 9) para indagar si los participantes han logrado entender que la fase de revisión y reescritura del texto escrito es uno de los momentos más fructíferos del aprendizaje, pues:

exige una lectura literal, inferencial y crítica, en tanto que pone en juego una serie de conocimientos adquiridos mediante la práctica, como mejorar el texto primigenio, solucionar problemas de escritura, evitar errores y desarrollar estrategias específicas para solventar problemas en los textos

$$
\text { (Álvarez Angulo, 2011, p. 272) }
$$

Los datos recabados muestran que los grupos han quedado satisfechos y recomendarían esta tarea, pues se alcanzan valores significativos en las opciones Totalmente de acuerdo y De acuerdo. No obstante, es necesario reconsiderar para futuras implementaciones algunas cuestiones de índole metodológica encaminadas a rebajar el porcentaje de estudiantes que al final del cuatrimestre todavía se muestran indiferentes ante tales prácticas. 


\section{REFERENCIAS}

Álvarez Angulo, T. (2011). Revisar y reescribir textos académicos en la formación del profesorado. Revista Complutense de Educación, 22(2), 269-294. http://dx.doi.org/10.5209/rev_RCED.2011.v22.n2.38493

Bazerman, C., y Russell, D. E. (Eds.). (1994). Landmark Essays on Writing Across the Curriculum (Vol. 6). Lawrence Erlbaum Associates.

Britton, J. (1970). Language and Learning. London: Allen Lane.

Bustos-Sánchez, A. (2009). Escritura colaborativa en línea. Un estudio preliminar orientado al análisis del proceso de co-autoría. RIED, 12(2), 33-55.

https://doi.org/10.5944/ried.2.12.900

Camps-Mundó, A., y Castelló-Badía, M. (2013). La escritura académica en la universidad. REDU. Revista de Docencia Universitaria, 11-1. https://doi.org/10.4995/redu.2013.5590

Carless, D. (2007). Learning-oriented assessment: conceptual bases and practical implications. Innovations in Education and Teaching International, 44(1), 57-66. https://doi.org/10.1080/14703290601081332

Carless, D. (2009). Learning-oriented assessment: Principles, Practice and a Project. En L. H. Meyer, S. Davidson, H. Anderson, R. Fletcher, P. M. Johnston, y M. Rees (Eds.), Tertiary Assessment \& Higher Education Student (pp. 79-90). Wellington: New Zealand: Ako Aotearoa.

Carless, D., Joughin, G., y Mok, M. (2006). Learning-oriented assessment: principles and practice. Assessment \& Evaluation in Higher Education, 31(4), 395-398. https://doi.org/10.1080/02602930600679043

Carlino, P. (2004). El proceso de escritura académica: cuatro dificultades de la enseñanza universitaria. Educere, 8(26), 321-327. Descargado de http://23118.psi.uba.ar/academica/cursos_actualizacion/recursos/carlino_cuatro.pdf

Castelló, M. (2014). Los retos actuales de la alfabetización académica: estado de la cuestión y últimas investigaciones. Enunciación, 19(2), 346-365.

https://doi.org/10.14483/10.14483/udistrital.jour.enunc.2014.2.a13

Cruz-Piñol, M. (2014). Veinte años de tecnologías y ELE. Reflexiones en torno a la enseñanza del español como lengua extranjera en la era de Internet. MarcoELE: Revista de Didáctica Español Lengua Extranjera(19). Descargado de https://marcoele.com/descargas/19/cruz-tecnologias.pdf

Erkens, G., Jaspers, J., Prangsma, M., y Kanselaar, G. (2005). Coordination processes in computer supported collaborative writing. Computers in Human Behavior, 21(3), 463-486. https://doi.org/10.1016/j.chb.2004.10.038

García-Chitiva, M. P., y Suárez-Guerrero, C. (2019). Estado de la investigación sobre la colaboración en Entornos Virtuales de Aprendizaje. Pixel-Bit. Revista de Medios y Educación(56), 169-191. https://dx.doi.org/10.12795/pixelbit.2019.i56.09

Gómez-Devís, M. B. (2015). La evaluación del aprendizaje en el aula universitaria: Una experiencia de innovación en la materia Lengua para Maestros. Opción(5), 963-982.

Gómez-Devis, M. B., Garcia-Raffi, J. V., y Saneleuterio, E. (2017). Educación lingüística y desarrollo de competencias en evaluación. Análisis de una experiencia en Magisterio. El Guiniguada, 26, 132-145. https://dx.doi.org/10.20420/elguiniguada.2017.142

Gómez-Devís, M. B., y Saneleuterio, E. (2015). E-evaluación orientada al aprendizaje: diseño para su implementación en la materia Lengua para maestros. RIAICES: Innovación en la Educación Superior desafíos y propuestas (pp. 319-326).

Gómez-Ruíz, M. A., y Quesada-Serra, V. (2017). Coevaluación o Evaluación Compartida en el Contexto Universitario: La Percepción del Alumnado de Primer Curso. Revista Iberoamericana de Evaluación Educativa, 10(2). https://dx.doi.org/10.15366/riee2017.10.2.001

Gómez-Ruiz, M. A., Rodríguez-Gómez, G., y Ibarra-Sáiz, M. S. (2013). Desarrollo de las competencias básicas de los estudiantes de Educación Superior mediante la 
e-Evaluación orientada al aprendizaje. RELIEVE - Revista Electrónica de Investigación y Evaluación Educativa, 19(1). https://dx.doi.org/10.7203/relieve.19.1.2457

Gros, B. (2015). The Dialogue Between Emerging Pedagogies and Emerging Technologies. En B. Gros, Kinshuk, M. Maina, et al. (Eds.), The Future of Ubiquitous Learning. Lecture Notes in Educational Technology (pp. 3-23). Berlin: Springer. https://doi.org/10.1007/978-3-662-47724-3_1

Hurtado-González, S. (2019). Estudio exploratorio de las técnicas y hábitos de composición textual en el nivel universitario. Lengua y Habla. Revista del Centro de Investigación y Atención Lingüística CIAL, 23, 15-29. Descargado de http://erevistas.saber.ula.ve/index.php/lenguayhabla/article/view/15660/21921926761

Ibarra, M., y Rodríguez-Gómez, S. (2010). Los procedimientos de evaluación como elementos de desarrollo de la función orientadora en la universidad. REOP. Revista Española de Orientación y Psicopedagogía, 21(2), 443-461.

https://doi.org/10.5944/reop.vol.21.num.2.2010.11558

Martínez-Lorca, M., y Zabala-Baños, C. (2015). Enseñando y aprendiendo a escribir en la universidad: Cuando los revisores son los compañeros. REDU. Revista de Docencia Universitaria, 13(3), 105-124. https://doi.org/10.4995/redu.2015.5422

Piscitelli, A. (2005). Internet, la imprenta del siglo XXI (and others, Ed.). Barcelona: GEDISA.

Ramos, G., Chiva, I., y Gómez-Devís, M. B. (2017). Las competencias básicas en la nueva generación de estudiantes universitarios: Una experiencia de Innovación. REDU. Revista de Docencia Universitaria, 15, 37-37. https://dx.doi.org/10.4995/redu.2017.5909

Reyes-García, C. I., Marchena-Gómez, R., y Sosa-Moreno, F. (2012). La evaluación orientada al aprendizaje y el diseño de proyectos docentes en la Universidad. El Guiniguada, 21, 117-132. Descargado de https://accedacris.ulpgc.es/bitstream/10553/12283/1/0235347_00021_0007.pdf

Rodríguez-Espinosa, H., Restrepo-Betancur, L. F., y Luna-Cabrera, G. C. (2016). Percepción del estudiantado sobre la evaluación del aprendizaje en la educación superior. Educare, 20(3), 1-17. https://dx.doi.org/10.15359/ree.20-3.18

Rodríguez-Gómez, G., y Ibarra-Saiz, M. S. (2015). Assessment as Learning and Empowerment: Towards Sustainable Learning in Higher Education. En M. Peris-Ortiz y J. M. Merigó-Lindahl (Eds.), Sustainable Learning inHigher Education. Developing Competencies for the Global Marketplace (pp. 1-20). http://dx.doi.org/10.1007/978-3-319-10804-9_1

Ruiz-Flores, M. (2009). Evaluación de la lengua escrita y dependencia de lo literal. Barcelona: Graó.

Russell, D. R. (1991). Writing in the Academic Disciplines, 1870-1990: A Curricular History. Carbondale: Southern Illinois University Press.

Russell, D. R., y Cortés, V. (2012). Academic and scientific texts: the same or different communities? En A. M. Castelló (Ed.), University Writing: Selves and Texts in Academic Societies (pp. 3-18). Emerald Group Publishing Limited. https://doi.org/10.1163/9781780523873_002

Saneleuterio, E., y García-Ramos, D. (2015). Diseño cooperativo para la optimización del aprendizaje lingüístico de futuros maestros. En and others (Ed.), RIAICES: Innovación en la Educación Superior desafíos y propuestas (pp. 425-432).

Scardamalia, M., y Bereiter, C. (2003). Knowledge building. En J. W. Guthrie (Ed.), Encyclopedia of Education (2nd ed.) (pp. 1370-1373). New York: Macmillan.

Scardamalia, M., y Bereiter, C. (2006). Knowledge building: Theory, pedagogy, and technology. En K. Sawyer (Ed.), Cambridge Handbook of the Learning Sciences (pp. 97-118). New York: Cambridge University Press.

Selfa-Sastre, M., y Gómez-Devís, M. B. (2019). Estudios científicos sobre la expresión escrita en el aprendizaje de segundas lenguas. Revista Internacional de Lenguas Extranjeras, 11, 1-17. https://doi.org/10.17345/rile11.2637

Solano-Córdoba, O. L. (2013). El aprendizaje combinado y el desarrollo de las habilidades 
requeridas para la comunicación escrita. Educare, 17(3), 293-313.

https://dx.doi.org/10.15359/ree.17-3.14

Sosa-Alonso, J. J., Santos-Vega, J. D., Vega-Navarro, A., y Marrero-Acosta, J. (2018).

Aprendiendo juntos con garantías de calidad: cooperación, socialización y evaluación en la enseñanza universitaria. En A. Vega-Navarro y D. Stendardi (Eds.), De la innovación imaginada a los procesos de cambio (pp. 45-66). Descargado de https://riull.ull.es/xmlui/bitstream/handle/915/9627/

3\%20Sosa\%20Alonso\%20Juan\%20Jos\%C3\%A9.pdf? sequence=1\&isAllowed=y

Tapia, S. M. (2017). La corrección de textos escritos en un profesorado virtual en Lengua y Literatura como experiencia didáctica para la formación docente. Virtualidad, Educación y Ciencia, 8(15), 162-172. Descargado de https://revistas.unc.edu.ar/index.php/vesc/article/view/18965

Vicent, M., Gonzálvez, C., Sanmartín, R., y Martínez-Monteagudo, M. C. (2018). Evaluación de la calidad de las estrategias metodológicas empleadas en la docencia universitaria: satisfacción del alumnado del Grado de Maestro. En R. Roig-Vila et al. (Eds.), El compromiso académico y social a través de la investigación e innovación educativas en la Enseñanza Superior (pp. 506-516). Barcelona: Octaedro. 